\title{
Evaluation of the Expression of c-MYC Protein during Liver Fibrosis Progression in Chronic Hepatitis Developed by Hepatitis C Virus Infected Patients
}

Terezinha Morato Bastos de Almeida ${ }^{1}$, Regina Maria Cubero Leitão², Flair José Carrilho ${ }^{3}$ Ana Maria Gonçalves da Silva ${ }^{3}$ and Shigueko Sonohara ${ }^{\star *}$

'Department of Radiology and Oncology, School of Medicine, University of São Paulo, São Paulo, Brazil

${ }^{2}$ Laboratory of Pathology Diagnóstika, São Paulo, Brazil

${ }^{3}$ Department of Gastroenterology, School of Medicine, University of São Paulo, São Paulo, Brazil

*Corresponding author: Shigueko Sonohara, Disciplina de Oncologia, Depto. de Radiologia e Oncologia, Faculdade de Medicina, Universidade de São Paulo, São Paulo, Brazil, Av. Dr. Arnaldo, 455, 4o. Andar, CEP 01246-903, Brazil, Tel: 0551130617470; Fax: 0551130826580; E-mail: shigueko@lim24.fm.usp.br

\begin{abstract}
Data on the onset and progression of fibrosis related to C-MYC protein expression in chronic hepatitis by $\mathrm{C}$ virus are scarce. In this work we evaluated by immunohistochemical method the expression of c-MYC protein in the liver parenchyma without fibrosis, and during its progression. Seventy-eight liver samples from Hepatitis $C$ virus infected patients with different degrees of fibrosis (FO to F4) were studied. Eleven healthy liver samples were included. All samples from chronic hepatitis were stained by hematoxylin-eosin method, and classified by METAVIR scoring system. The expression of c-MYC protein was evaluated in 1000 hepatocytes from each parenchyma area by immunohistochemistry. Results showed that C-MYC expression in hepatocytes from fibrosis samples (F1-F4) was higher, and statistically significant $(\mathrm{P}<0.05)$ when compared with the control group. The same results were obtained when comparing F4 vs F0; F1; F2 and F3. In contrast, the comparisons between F0 vs control; F1 vs F2; F1 vs F3; and F2 vs F3 did not present significant differences $(P>0.05)$. In conclusion, our study showed that $c-M Y C$ protein is present in the initial degrees of fibrosis, but over-expressed in F4. This fact suggests that alteration of C-MYC expression may induce genetic instability, deregulating the cell cycle and/or apoptosis, which might contribute to progression of fibrosis and ultimately predisposing to the development of hepatocellular carcinoma.
\end{abstract}

Keywords: Chronic hepatitis C; Fibrosis levels; c-MYC protein

\section{Abbreviations:}

HCV: Hepatitis C Virus; CH: Chronic Hepatitis; LC: Liver Cirrhosis; RN: Regenerative Nodule; HCC: Hepatocellular Carcinoma; NP: Normal Parenchyma

\section{Introduction}

c-MYC protein plays an important role in cell cycle regulation, apoptosis, differentiation, metabolism, genomic instability and angiogenesis [1-3]. The involvement of c-MYC protein expression in liver tissue regeneration and chemically induced hepatocarcinogenesis was early suggested [4-8]. Significantly high expression of c-MYC gene was found in hepatocellular carcinoma (HCC), and some of liver samples from cirrhotic patients infected by hepatitis B virus (HBV), as compared to normal liver [9]. Also, significantly high expression of cmyc m-RNA was found in early HCC [10]. Progressive increase of cMYC m-RNA and its protein was detected during the stages of HCC development [11]. The level of this protein was shown in poorly differentiated tumors, and it was significantly higher as compared to well differentiated tumors, showing that over expression of c-MYC was correlated to the histological differentiation of HCC. c-MYC protein was found to be expressed in HCC, liver cirrhosis (LC), and chronic hepatitis $(\mathrm{CH})$ of HBV [12] and hepatitis $\mathrm{C}$ virus (HCV) infected tissues [13] at higher levels than normal liver, suggesting the importance this protein has also in the precancerous phase [14].

In spite of these reports, a detailed study correlating c-MYC protein with $\mathrm{CH}$ development toward LC in $\mathrm{HCV}$-infected patients are scarce. Thus, in this study we assessed the immunoreactivity of c-MYC protein in $\mathrm{CH}$ during the progression of fibrosis ( $\mathrm{F} 1$ to $\mathrm{F} 4$ ), on a large number of cases, comparing these data to that obtained from F0 and normal liver.

\section{Materials and Methods}

\section{Tissue samples}

Liver tissue samples were obtained from Departamento de Patologia, Faculdade de Medicina da Universidade de São Paulo, and from Laboratório de Anatomia Patológica Diagnóstika, São Paulo. Our study comprised of 78 male and female patients, all of them with a history of chronic hepatitis by $\mathrm{HCV}(\mathrm{CH} / \mathrm{HCV})$, classified into the following groups: F0 (11); F1 (13); F2 (18); F3 (13); F4 (23) and 11 control individuals. Fibrosis progressive stages (F0 to F4) of the parenchyma with chronic hepatitis were classified according to both the METAVIR System [15], and International Working Party [16]. Stages are: F0, without fibrosis; F1- mild fibrosis; F2- moderate fibrosis; F3- severe fibrosis and F4- cirrhosis in regenerative nodules (RNs) without atypia. The hepatic normal parenchyma was randomly chosen. According to anamnesis, none of the patients had been previously exposed to any kind of other etiological agent, including those known as mutagenic.

\section{Procedures}

The $\mathrm{CH} / \mathrm{HCV}$ samples were fixed in $10 \%$ formalin buffer and embedded in paraffin blocks, following the standard procedure. Blocks were cut into 3-5 $\mu$ m-thick sections. All liver samples were placed on slides previously prepared with 3-aminopropil-trietoxysilane (Sigma). 
Citation: de Almeida TMB, Leitao RMC, Carrilho FJ, da Silva AMG, Sonohara S (2014) Evaluation of the Expression of c-MYC Protein during Liver Fibrosis Progression in Chronic Hepatitis Developed by Hepatitis C Virus Infected Patients. J Carcinog Mutagen 5: 182. doi: $10.4172 / 2157-2518.1000182$

Page 2 of 5

In each slide, areas were selected according to histopathological analysis after staining by the hematoxylin-eosin method.

\section{Immunohistochemistry of c-MYC protein}

Formalin-fixed and paraffin embedded tissue sections were incubated at $56^{\circ} \mathrm{C}$ for $16 \mathrm{~h}$, and then immersed twice in xylene at room temperature. Subsequently, slides were rehydrated in a graded alcohol series and distilled water. Antigen retrieval was performed in $0.01 \mathrm{M}$ citrate buffer ( $\mathrm{pH} \mathrm{6)}$ and incubation in a microwave oven for 45 minutes at high temperature. After cooling, they were rinsed with distilled water. To block the endogenous peroxidase, the slides were immersed in $1 \% \mathrm{H}_{2} \mathrm{O}_{2} /$ methanol for 10 minutes. Subsequently, the sections were rinsed with distilled water, and blocked in $6 \%$ skimmed milk diluted in PBS for 30 minutes, followed by three rinses with 0.05 $\mathrm{M}$ Tris $/ \mathrm{HCl} / 0.3 \mathrm{M} \mathrm{NaCl} / 0.1 \%$ Tween 20 . The sections were incubated overnight at $4^{\circ} \mathrm{C}$ with monoclonal antibody c-MYC (NCL-MYC-C) clone 9E10 (Dako) at a dilution of 1:200 in 0.1\% BSA/PBS. Then, the sections were rinsed with $0.05 \mathrm{M}$ Tris/ $/ \mathrm{HCl} / 0.3 \mathrm{M} \mathrm{NaCl} / 0.1 \%$ Tween 20 for 5 minutes each, and incubated with secondary antibody (Dako Envision System HRPDAB) for 30 minutes at room temperature, followed by 3 rinses with $0.05 \mathrm{M}$ Tris/HCl/0.3M NaCl/0.1\% Tween 20 . For the visualization of bound antibody, $0.04 \% 3,3^{\prime}$ diaminobenzidine tetrahydrochloride (Sigma Chemical Co) in PBS was added, followed by the addition of $1 \% \mathrm{H}_{2} \mathrm{O}_{2} / \mathrm{PBS}$ for about one minute, and rinsings with distilled water. After counterstaining with Carazzi`s hematoxylin and rinsing in distilled water, the sections were dehydrated in a graded alcohol series. Section transparency was carried out by xylene baths (three times), and mounted for viewing with a microscope. The negative controls were submitted to the same process as above, but the primary antibody was replaced with $0.1 \%$ BSA/PBS.

\section{Immunohistochemical analysis}

For this purpose 1000 hepatocytes per parenchymal area were evaluated for c-MYC protein positiveness, that is, the brown color of the nuclei, after the immunohistochemical reaction. The predominant positive pattern of all cells was analyzed and its representation was based on c-MYC staining grade, as follows: + (weak); ++ (moderate); + ++ (strong). The values of c-MYC positive nuclei were expressed as a percent of the total of the hepatocytes evaluated.

\section{Photographic documentation}

Expression of c-MYC protein was documented by means of an Olympus BX- 51 epifluorescence photomicroscope with immersion lens, fitted with optovars (1.25x, 1.6x and 2.0x).

\section{Statistical analysis}

For the statistical analysis, we considered all positive hepatocytes, independent on the intensity grade of c-MYC (weak, moderate or strong) of all cases from each category of liver fibrosis. Differences among groups were checked through non parametric analysis of variance (Kruskal-Wallis test) followed by multiple comparison tests, in order to locate those comparisons responsible for the significance of the test [17]. In all cases, differences were considered statistically significant when $\mathrm{P}$ was $\leq 0.05$.

\section{Results}

Our results showed that the expression of c-MYC protein in hepatocytes was predominantly nuclear in all analyzed cases of groups F0, F1, F2, F3, and F4, including 45\% of the control group. The variation in the intensity of staining of the c-MYC protein, was observed to be weak $(+)$, moderate $(++)$, and strong $(+++)$, as can be seen in figures la-1f. Intensity of staining was linearly correlated to intensity of expression of the c-MYC protein. In F0 cases $73 \%$ presented weak expression in relation to F1, F2, F3, F4 groups. Strong staining was evidenced in $4 / 18$ cases of F2 (22\%), in $2 / 13$ cases of F3 (15\%), and in $6 / 23$ cases of the F4 (26\%). Moderated staining of cMYC protein was observed in F4 group, $13 / 23$ cases (56\%), F3 with $6 / 13$ cases (46\%), F2 with $8 / 18$ cases (44\%), F1 with $2 / 13$ cases $(15 \%)$, and F0 with $4 / 11$ cases (36\%) (Table 1).

\begin{tabular}{|l|l|l|l|l|l|}
\hline Groups & $\begin{array}{l}\text { Number } \\
\text { of cases }\end{array}$ & $\begin{array}{l}\text { Positive } \\
\text { Expression } \\
(\%)\end{array}$ & \multicolumn{4}{|l|}{ Intensity of staining (\%) } \\
\hline & & & + & ++ & +++ \\
\hline F0 & 11 & $8(73)$ & $8(73)$ & $4(36)$ & 0 \\
\hline F1 & 13 & $13(100)$ & $13(100)$ & $2(15)$ & 0 \\
\hline F2 & 18 & $18(100)$ & $17(94)$ & $8(44)$ & $4(22)$ \\
\hline F3 & 13 & $13(100)$ & $13(100)$ & $6(46)$ & $2(15)$ \\
\hline F4 & 23 & $23(100)$ & $22(95)$ & $13(56)$ & $6(26)$ \\
\hline NP & 11 & $5(45)$ & $5(45)$ & 0 & 0 \\
\hline
\end{tabular}

Table 1: Expression of c-MYC protein in hepatocytes of chronic C hepatitis cases (fibrosis levels F0 to F4) and normal parenchyma (NP).

The proportion of c-MYC protein positivity in hepatocytes are as follows: diffuse, F4 (62.76\%); F3 (31.74\%); F2 (20.34\%); F1 (26.15\%); intermediate: F4 (6.28\%); F3 (1.22\%); F2 (2.26\%); F1 (1.75\%); focal: F4 (0.53\%); F3 (0.49\%); F2 (0.07\%); F1 (0\%). The cases of F0 group presented $3.59 \%$ of the cells with diffuse staining of c-MYC protein, $0.98 \%$ of them with intermediate and focal staining $(0 \%)$. In the control group $1.33 \%$ of the cells presented only diffuse expression (Table 2).

\begin{tabular}{|l|l|l|l|l|}
\hline \multirow{2}{*}{ Groups } & \multirow{2}{*}{ Negative } & \multicolumn{3}{l}{ Positive of stanining } \\
\cline { 3 - 5 } & & \multicolumn{2}{|l|}{ Intensity of Staining (\%) } \\
\cline { 3 - 5 } & & + & ++ & +++ \\
\hline F0 & 95.43 & 3.59 & 0.98 & 0 \\
\hline F1 & 72.10 & 26.15 & 1.75 & 0 \\
\hline F2 & 77.33 & 20.34 & 2.26 & 0.07 \\
\hline F3 & 66.55 & 31.74 & 1.22 & 0.49 \\
\hline F4 & 30.43 & 62.76 & 6.28 & 0.53 \\
\hline NP & 98.67 & 1.33 & 01 & 0 \\
\hline
\end{tabular}

Table 2: Percentage of heaptovytes with positive staining for c-MYC protein of chronic C hepatitis groups (F0 to F4) and normal parenchyma (Contorl group). 
Citation: de Almeida TMB, Leitao RMC, Carrilho FJ, da Silva AMG, Sonohara S (2014) Evaluation of the Expression of c-MYC Protein during Liver Fibrosis Progression in Chronic Hepatitis Developed by Hepatitis C Virus Infected Patients. J Carcinog Mutagen 5: 182. doi: $10.4172 / 2157-2518.1000182$

Page 3 of 5

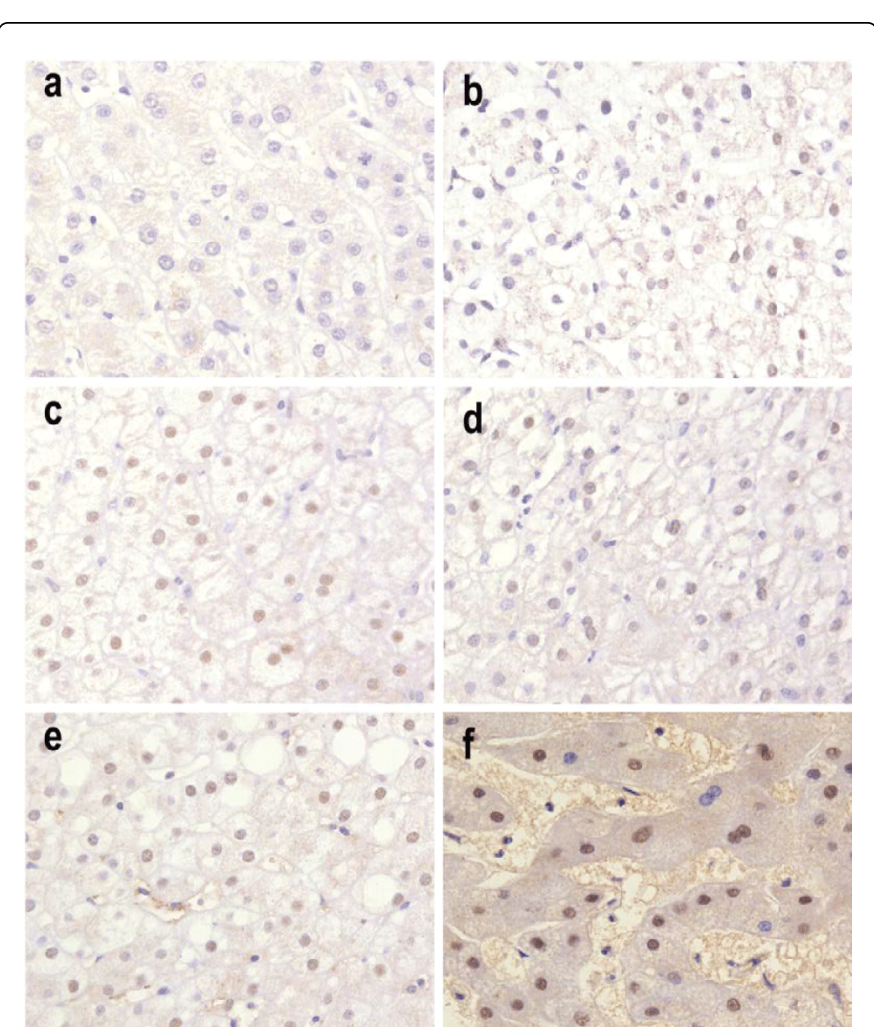

Figure 1: Tissue sections showing the expression of c-MYC protein (nuclear staining) in liver parenchyma with $\mathrm{CH}$ in different fibrosis levels, which appears in brown colour. All pictures have magnification of 400x. a- there was no positive immunostaining of c-Myc (blue colour) in hepatocytes from NP; b- F0: cells with weak positive expression $(+)$; c, d, and e- F1, F2, F3, respectively, as follows: cells with weak $(+)$, with moderate $(++)$, and $\mathrm{f}: \mathrm{F} 4$, shows weak, moderate, and strong $(+++)$ positive expression of c-MYC.

The frequencies of hepatocytes expressing the protein c-MYC was significantly higher in F1, F2, F3 and F4 as compared to NP (control), as is depicted in Table 3, and Figure 2. In contrast, the frequency in F0 hepatocytes and in control group was not different $(\mathrm{p}>0.05)$. F0 group presented significantly lower frequency of c-MYC expression when compared to F1, F2, F3, F4 groups. The parenchyma of $\mathrm{CH}$ in the first level of fibrosis (F1) presented hepatocytes with c-MYC expression lower than that of F4, but the same level than that of F2 and F3. Expression levels in F2 and F3 are lower than that observed in F4 group (Table 1).

\section{Discussion}

The expression of a cellular oncogene such as c-MYC during fibrosis progression in liver parenchyma of patients suffering chronic hepatitis $(\mathrm{CH})$ developed after $\mathrm{HCV}$ infection, was investigated in details. Our immunohistochemical data showed the presence of cMYC protein, and variations of its expression in $\mathrm{CH}$ hepatocytes from $\mathrm{HCV}$-infected patients without fibrosis (F0), as well as in those with different levels of fibrosis (F1, F2, F3, and F4). The positive staining of c-MYC protein was predominantly nuclear in hepatocytes from all $\mathrm{CH}$ groups (F0-F4), and was more intense in F1, F2, F3, and F4. These results are in agreement with the investigators that localized c-MYC protein in LC and normal parenchyma in paraffin embedded samples $[11,12]$. Some authors have also detected c-MYC expression in the cytoplasm. However, fixation artifacts may not be discarded $[13,18]$.

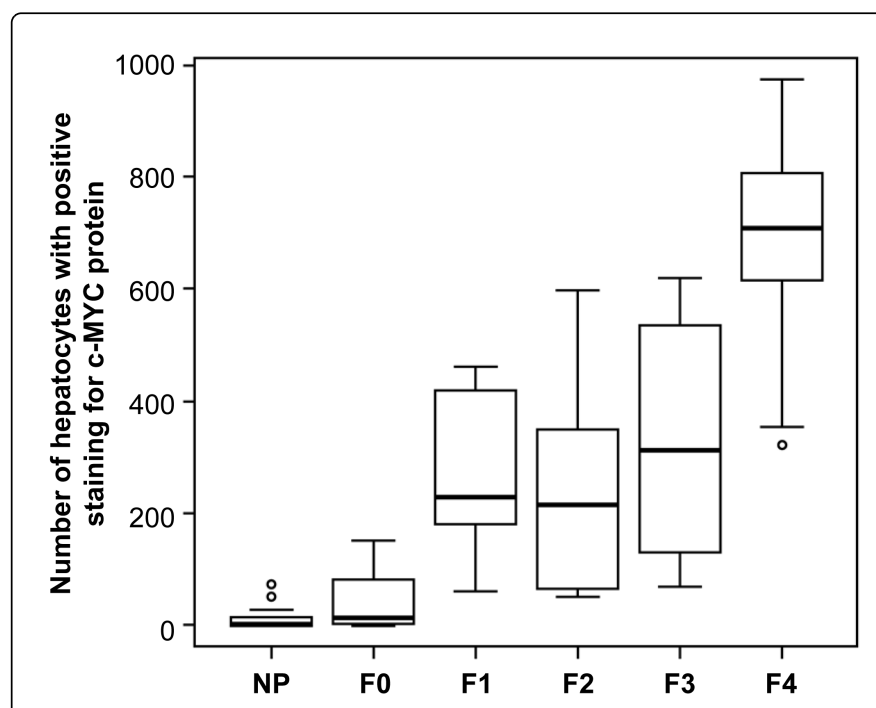

Figure 2: Expression of c-MYC protein on the progression of fibrosis levels F0 to F4 and normal parenchyma (NP). Boxes indicate medians, whiskers and minimal and maximum values, extending to 1.5 times the interquartile range; circles represent outliers.

\begin{tabular}{|l|l|l|l|l|l|}
\hline Groups & NP & F0 & F1 & F2 & F3 \\
\hline F0 & 0.6776 & - & - & - & - \\
\hline F1 & $<0.0001$ & $<0.0001$ & - & - & - \\
\hline F2 & $<0.0001$ & $<0.0009$ & 0.9769 & - & - \\
\hline F3 & $<0.0001$ & $<0.0001$ & 0.9964 & 0.5513 & - \\
\hline F4 & $<0.0001$ & $<0.0001$ & $<0.0001$ & $<0.0001$ & $<0.0001$ \\
\hline
\end{tabular}

Table 3: $\mathrm{P}$ values calculated from multiple comparisions of expression c-MYC protein on the progression of fibrosis levels F0 to F4 and normal parenchyma (NP)Significance set at $\mathrm{p}<0.05$.

In spite of absence of fibrosis, the F0 group presented expression of c-MYC protein, in approximately $4.5 \%$ of hepatocytes. This result suggests that the presence of c-MYC protein expression in $\mathrm{CH}$ can emerge at the start of inflammatory process, increasing liver tissue regeneration, due to $\mathrm{HCV}$ infection. In particular, the $\mathrm{HCV}$ core protein causes perturbation of cell cycle progression in a human hepatoblastoma transfected cells [19]. Similarly in transgenic mouse model [20] it has been attributed to HCV core protein the role of modulation of intracellular signaling pathways conferring an advantage for cell proliferation to hepatocytes. In the mitotic cell cycle deregulation, the $\mathrm{HCV}$ core protein may be directly implicated in the induction of the chromosome instability in hepatocytes [21]. Interestingly, it has also been consistently observed that HCV core is important in activation of c-MYC expression. The HCV core protein promotes increased c-MYC expression, in both liver from HCVinfected patients and in mice model expressing the entire HCV ORF in a liver specific manner [22]. Thus, the c-MYC protein expression early 
Citation: de Almeida TMB, Leitao RMC, Carrilho FJ, da Silva AMG, Sonohara S (2014) Evaluation of the Expression of c-MYC Protein during Liver Fibrosis Progression in Chronic Hepatitis Developed by Hepatitis C Virus Infected Patients. J Carcinog Mutagen 5: 182. doi: $10.4172 / 2157-2518.1000182$

Page 4 of 5

in $\mathrm{CH}-\mathrm{F} 0$ may point toward the gradual deterioration of the liver parenchyma in the progression of $\mathrm{CH}$ to $\mathrm{LC}$.

In our study, the expression of c-MYC protein was significantly higher in hepatocytes during fibrosis progression (F1 to F4). Levels of c-MYC protein from F1 to F3 are maintained up to F4 stage when they are perpetuated in a significantly higher level. The expression of the cMYC gene may indicate a premalignant condition which lead $\mathrm{CH}$ toward HCC after HCV infection. This fact suggests that the damage caused by chronic infection by HCV should be intensified during the development of fibrosis. Increased expression of c-MYC protein was reported in liver samples of patients with $\mathrm{CH}$ and LC infected with Hepatitis B virus [12], and HCV [13], however these authors did not correlate their data to all the stages of fibrosis development. Recently, evidences that c-MYC is over-expressed in hepatocytes during hepatic fibrogenesis were described in human and animal model [23].

Our data, as well as results from other authors, strongly suggest that alteration of c-MYC protein expression is linked to several hepatic alterations which eventually predispose hepatocytes to genetic instability. Some studies have indicated that the deregulation of the cMYC gene expression, generates genomic instability due to an onset of DNA amplification [24-26]. Our previous results [27,28] showed that hepatocytes, from $\mathrm{CH}$ at different levels of liver fibrosis (F1-F4), presented cytogenetic instability, detected by micronuclei formation, in relation to normal liver parenchyma. In our recent data [29] obtained by using FISH technique, revealed c-MYC gene amplification in hepatocytes from the same area of ordinary regenerative nodules (RNs) from LC cases (F4). This event is accompanied by increased expression of the c-MYC protein as the present study demonstrates. Although, the relationship between altered c-MYC protein expression, and gene amplification is still unclear in liver tissue, the results obtained in our investigation can point to the role of c-MYC gene in the transformation of hepatocytes from ordinary RNs during the hepatocarcinogenesis.

Together, these results suggest that assessing levels of c-MYC protein in $\mathrm{CH}$ during fibrosis stages may be useful to establish patient prognosis. These levels are apparently early markers of LC and cancer development, and may be seen as potential target for therapy.

\section{Acknowledgments}

This work was supported by grants from Fundação de Amparo à Pesquisa do Estado de São Paulo (FAPESP), Sense Eletrônica Ltda, Centro de Estudos em Gastroenterologia - Agostinho Betarello, and Alves de Queiróz Family Fund for Research. We are grateful to Helen Susan Stavros Castelhano, and Helena Khouri for English language revision; João Ítalo Dias França for statistical analysis revision, and Maria Cristina Granero Grandal for figure editing.

\section{References}

1. Eilers M, Eisenman RN (2008) Myc's broad reach. Genes Dev 22: 2755-2766.

2. Prochownik EV, Li Y (2007) The ever expanding role for c-Myc in promoting genomic instability. Cell Cycle 6: 1024-1029.

3. Patel JH, Loboda AP, Showe MK, Showe LC, McMahon SB (2004) Analysis of genomic targets reveals complex functions of MYC. Nat Rev Cancer 4: 562-568.

4. Morello D, Fitzgerald MJ, Babinet C, Fausto N (1990) c-myc, c-fos, and $\mathrm{c}$-jun regulation in the regenerating livers of normal and $\mathrm{H}-2 \mathrm{~K} / \mathrm{c}-\mathrm{myc}$ transgenic mice. Mol Cell Biol 10: 3185-3193.
5. Su TS, Lin LH, Lui WY, Chang CM, Chou CK, et al. (1985) Expression of c-myc gene in human hepatoma. Biochem Biophys Res Commun 132: 264-268.

6. Thompson NL, Mead JE, Braun L, Goyette M, Shank PR, et al. (1986) Sequential protooncogene expression during rat liver regeneration. Cancer Res 46: 3111-3117.

7. Zhang XK, Wang Z, Lee A, Huang DP, Chiu JF (1988) Differential expression of cellular oncogenes during rat liver development. Cancer Lett 41: 147-155.

8. Buendia MA, Bourre L, Cairo S (2012) Myc target miRs and liver cancer: small molecules to get Myc sick. Gastroenterology 142: 214-218.

9. Zhang X-K, Huang DP, Qiu D-K, Chiu J-F (1990). The expression of cmyc and c-N-ras in human cirrhotic livers, hepatocellular carcinomas and liver tissue surrounding the tumors. Oncogene 5: 909-914.

10. Kaposi-Novak P, Libbrecht L, Woo HG, Lee YH, Sears NC, et al. (2009) Central role of $\mathrm{c}$-Myc during malignant conversion in human hepatocarcinogenesis. Cancer Res 69: 2775-2782.

11. Gan FY, Gesell MS, Alousi M, Luk GD (1993) Analysis of ODC and cmyc gene expression in hepatocellular carcinoma by in situ hybridization and immunohistochemistry. J Histochem Cytochem 41: 1185-1196.

12. Chan KL, Guan XY, Ng IO (2004) High-throughput tissue microarray analysis of c-myc activation in chronic liver diseases and hepatocellular carcinoma. Hum Pathol 35: 1324-1331.

13. El-Bassionni A, Nosseir M, Zoheiry M, El-Ahwany E, Ghali A et al. (2006). Immunohistochemical expression of CD95 (Fas), c-myc and epidermal growth factor in hepatitis $\mathrm{C}$ virus infection, cirrhotic liver disease and hepatocellular carcinoma. APMIS 114: 420-427.

14. Feitelson MA (2004) c-myc overexpression in hepatocarcinogenesis. Hum Pathol 35: 1299-1302.

15. [No authors listed] (1994) Intraobserver and interobserver variations in liver biopsy interpretation in patients with chronic hepatitis $\mathrm{C}$. The French METAVIR Cooperative Study Group.Hepatology 20: 15-20.

16. International Working Party (1995) Terminology of nodular hepatocellular lesions. Hepatology 22: 983-993.

17. Dean CB1, Nielsen JD (2007) Generalized linear mixed models: a review and some extensions. Lifetime Data Anal 13: 497-512.

18. Moghul A, Lin L, Beedle A, Kanbour-Shakir A, DeFrances MC, et al. (1994) Modulation of c-MET proto-oncogene (HGF receptor) mRNA abundance by cytokines and hormones: evidence for rapid decay of the 8 kb c-MET transcript Oncogene 9: 2045-2052.

19. Ruggieri A, Murdolo M, Harada T, Miyamura T, Rapicetta M (2004) Cell cycle perturbation in a human hepatoblastoma cell line constitutively expressing Hepatitis C virus core protein. Arch Virol 149: 61-74.

20. Koike K, Tsutsumi T, Miyoshi H, Shinzawa S, Shintani Y, et al. (2008) Molecular basis for the synergy between alcohol and hepatitis $\mathrm{C}$ virus in hepatocarcinogenesis. J Gastroenterol Hepatol 23 Suppl 1: S87-91.

21. Baek KH, Park HY, Kang CM, Kim SJ, Jeong SJ, et al. (2006) Overexpression of hepatitis C virus NS5A protein induces chromosome instability via mitotic cell cycle dysregulation. J Mol Biol 359: 22-34.

22. Higgs MR, Lerat H, Pawlotsky JM (2013) Hepatitis C virus-induced activation of $\hat{\mathrm{I}}^{2}$-catenin promotes c-Myc expression and a cascade of procarcinogenetic events. Oncogene 32: 4683-4693.

23. Nevzorova YA, Hu W, Cubero FJ, Haas U, Freimuth J, et al. (2013) Overexpression of c-myc in hepatocytes promotes activation of hepatic stellate cells and facilitates the onset of liver fibrosis. Biochim Biophys Acta 1832: 1765-1775.

24. Mai S, Mushinski JF (2003) c-Myc-induced genomic instability. J Environ Pathol Toxicol Oncol 22: 179-199.

25. Kuttler F, Mai S (2006) c-Myc, Genomic Instability and Disease. Genome Dyn 1: 171-190.

26. Prochownik EV (2008) c-Myc: linking transformation and genomic instability. Curr Mol Med 8: 446-458.

27. de Almeida TMB, Leitao RC, Andrade JD, Becak W, Carrilho FJ et al. (2004). Detection of micronuclei formation and nuclear anomalies in 
Citation: de Almeida TMB, Leitao RMC, Carrilho FJ, da Silva AMG, Sonohara S (2014) Evaluation of the Expression of c-MYC Protein during Liver Fibrosis Progression in Chronic Hepatitis Developed by Hepatitis C Virus Infected Patients. J Carcinog Mutagen 5: 182. doi: 10.4172/2157-2518.1000182

Page 5 of 5

regenerative nodules of human cirrhotic livers and relationship to hepatocellular carcinoma. Cancer Genet Cytogenet 150: 16-21.

28. de Almeida TM, Leitão RM, Carrilho FJ, Sonohara S (2010) Micronuclei formation in liver fibrosis samples from patients infected by hepatitis $\mathrm{C}$ virus. Genet Mol Biol 33: 418-421.
29. de Almeida TMB, Leitao RMC, Yoshimoto M, Andrade JAD, Becak W, et al. (2012). Detection of c-MYC gene in micronucleated hepatocytes from regenerative cirrhotic nodules and hepatocellular carcinoma of hepatitis $\mathrm{C}$ virus infected patients. J Carcinog Mutagen 3: 130. 